\title{
Leveraging Loosely-Tagged Images and Inter-Object Correlations for Tag Recommendation
}

\author{
Yi Shen \\ Dept of Computer Science \\ UNC-Charlotte \\ Charlotte, NC 28223, USA \\ yshen9@uncc.edu
}

\author{
Jianping Fan \\ Dept of Computer Science \\ UNC-Charlotte \\ Charlotte, NC 28223, USA \\ jfan@uncc.edu
}

\begin{abstract}
Large-scale loosely-tagged images (i.e., multiple object tags are given loosely at the image level) are available on Internet, and it is very attractive to leverage such loosely-tagged images for automatic image annotation applications. In this paper, a multi-task structured SVM algorithm is developed to leverage both the inter-object correlations and the loosely-tagged images for achieving more effective training of a large number of inter-related object classifiers. To leverage the loosely-tagged images for object classifier training, each loosely-tagged image is partitioned into a set of image instances (image regions) and a multiple instance learning algorithm is developed for instance label identification by automatically identifying the correspondences between multiple tags (given at the image level) and the image instances. An object correlation network is constructed for characterizing the inter-object correlations explicitly and identifying the inter-related learning tasks automatically. To enhance the discrimination power of a large number of inter-related object classifiers, a multi-task structured SVM algorithm is developed to model the inter-task relatedness more precisely and leverage the inter-object correlations for classifier training. Our experiments on a large number of inter-related object classes have provided very positive results.
\end{abstract}

Categories and Subject Descriptors

I.4.8 [Image Processing and Computer Vision]: Scene Analysis-object recognition.

General Terms

Algorithms, Measurement, Experimentation

\section{Keywords}

Multi-task structured SVM, object correlation network, looselytagged images, multiple instance learning.

\section{INTRODUCTION}

For many image understanding tasks, such as object detection and scene recognition, machine learning techniques are usually involved to learn the classifiers from large amounts

Permission to make digital or hard copies of all or part of this work for personal or classroom use is granted without fee provided that copies are not made or distributed for profit or commercial advantage and that copies bear this notice and the full citation on the first page. To copy otherwise, to republish, to post on servers or to redistribute to lists, requires prior specific permission and/or a fee.

MM'10, October 25-29, 2010, Firenze, Italy.

Copyright 2010 ACM 978-1-60558-933-6/10/10 ...\$10.00. of training images and the ground-truth labels for the training images are usually provided by professionals. Because it is labor intensive to hire professionals for labeling large amounts of training images, the sizes of such professionallylabeled image sets tend to be small. As a result, the classifiers, which are learned from a small set of the professionallylabeled training images, may hardly be generalizable. To achieve more reliable classifier training, the size of the labeled training images must be large due to: (1) the number of object classes could be very large; and (2) the learning complexity for some object classes could be very high because they may have large intra-class visual diversity and large inter-class visual similarity (i.e., visual ambiguity).

One way for us to collect large-scale training images is to leverage the advantages of collaborative image tagging systems (i.e., leveraging the collaborative efforts of a large population of Internet users) [1-2]. To differentiate such collaboratively-tagged images from the professionally-labeled images, we call them as loosely-tagged images because multiple object tags are loosely given at the image level rather than at the object level (i.e., without providing the exact object locations in the images).

Large-scale loosely-tagged images can have multiple advantages: (1) they can represent various visual properties of the object classes more sufficiently; (2) they can be obtained easily by leveraging the collaborative efforts of large numbers of Internet users, our fundamental belief is that a large group of Internet users with diverse backgrounds can do better job than a small team of professionals as illustrated by wikipedia; (3) both their tags and their visual properties are diverse, thus they can give a real-world point of departure for object detection and scene recognition. Therefore, it is very attractive to develop new learning frameworks which are able to leverage the loosely-tagged images for object classifier training.

It is not a trivial task to leverage the loosely-tagged images for object classifier training because they may seriously suffer from the critical issue of tag uncertainty (i.e., loose tags and multiple tags). In a collaborative image tagging space, the object tags are usually given at the image level (i.e., loose tags without providing the exact locations for the objects in the images) because providing the tags at the image level is far less time consuming. On the other hand, one single image may be associated with multiple tags (i.e., multi-label image) because it may contain multiple objects. When multiple tags are frequently used to tag the images jointly, they should have strong correlations (i.e., inter-tag correlations), but how to leverage such inter-tag (inter-object) correlations 


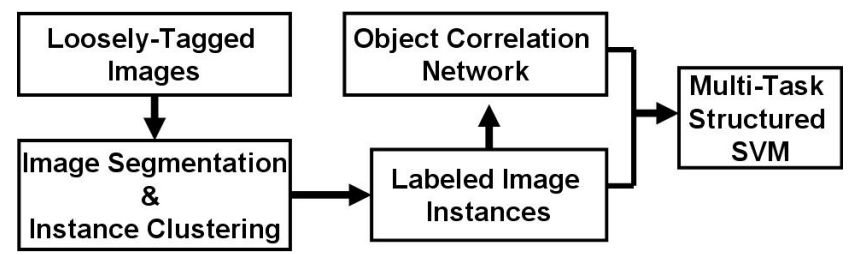

Figure 1: The flowchart of our multi-task structured SVM scheme for inter-related classifier training.

for object classifier training is still an open issue for the multimedia research community [13-18]. Ignoring the interobject correlations may result in the object classifiers with low discrimination power.

To leverage both the loosely-tagged images and the interobject correlations for object classifier training, it is very attractive to develop new frameworks for: (a) ambiguous image representation which can transform each loosely-tagged image into bags of instances and express its semantics ambiguity (i.e., multiple tags) explicitly in the instance space; (b) identifying the instance labels automatically when the tags are provided only at the image level (i.e., loose tags); and (c) structured learning for exploiting the inter-object correlations to achieve more effective learning of a large number of inter-related object classifiers.

As shown in Fig. 1, a multi-task structured SVM algorithm is developed to leverage both the loosely-tagged images and the inter-object correlations to achieve more accurate training of a large number of inter-related object classifiers. This paper is organized as follows. Section 2 reviews the related work briefly; Section 3 presents a multiple instance learning algorithm to automatically identify the labels for the relevant image instances in the positive bags; Section 4 introduces an interesting approach to construct an object correlation network for identifying the interrelated learning tasks automatically; Section 5 presents our multi-task structured SVM algorithm for inter-related classifier training; Section 6 describes our work on algorithm evaluation; We conclude in Section 7.

\section{RELATED WORK}

Some pioneering work have been done on multiple instance learning [7-10]. Chen et al. [10] have developed an interesting approach called MILES to enable region-based image annotation when the labels are available only at the image level. Vijayanarasimhan et al. have developed a multi-label multiple instance learning approach to achieve more effective learning from the loosely-labeled images [7]. Zhang et al. [8] and Maron et al. [9] have incorporated multiple instance learning (MIL) techniques to learn the object detectors from the loosely-labeled images.

In order to incorporate multi-label images for classifier training, some pioneering work have been done by dividing multi-label learning into a set of binary classification problems or transforming multi-label learning into a label ranking problem [11-12]. Boutell et al. have addressed the issue of multi-label image annotation by learning a set of binary classifiers [11]. Zhou et al. [12] and Zha et al. [22] have integrating multiple instance learning with multi-label learning for scene classification by exploiting the inter-label correlations at the label space. Because classifier training is performed in the feature space rather than in the label space, it is very attractive to develop new algorithms that can directly model the inter-object correlations in the feature space.

Multi-task learning has widely been studied by exploiting the correlations between multiple learning tasks [13-18]. Torralba et al. [17] have developed a novel JointBoost algorithm to support multi-task learning. Jiang et al. [18] have extended such JointBoost algorithm for multi-class concept detection by sharing common kernels. The boosting algorithm could be very sensitive to data noise, thus it cannot directly be used to leverage the loosely-tagged images with large tag uncertainty for classifier training. Kumar et al. have proposed Discriminative Random Fields (DRF) to exploit the inter-patch correlations for object detection [15]. Yang et al. [16] have recently extended the DRF technique for image/video concept detection. Fan et al. have constructed concept ontology for identifying the inter-related learning tasks in the concept space and achieving hierarchical training of a large number of inter-related image classifiers [13].

The statistical rules, such as object co-occurrence contexts, have been derived from large-scale image collections for supporting context-driven object detection and some pioneering work have been done recently [19-22]. Qi et al. [21], Tang et al. [19] and Liu et al. [20] have exploited the correlations between the image/video concepts to enhance automatic image/video annotation, and some interesting statistical models have been developed to leverage such inter-concept contexts for concept classifier training.

In order to leverage both the inter-object correlations and the inter-task relatedness for classifier training, a multi-task structured SVM algorithm is developed in this paper: (1) An object correlation network is constructed for representing the inter-object correlations explicitly and providing a good environment to identify the inter-related learning tasks directly in the feature space rather than in the label space. (2) A multiple instance learning algorithm is developed for identifying the labels of the image instances automatically when the labels are only available at the image level. (3) A multitask structured SVM algorithm is developed by incorporating the object correlation network, structured SVM [23-24] and multi-task learning [13-14, 17-18] to model the inter-task relatedness more precisely and leverage the inter-object correlations for training a large number of inter-related object classifiers jointly.

\section{MULTIPLE INSTANCE LEARNING}

To address the issues of loose tags and multiple tags simultaneously, two bags of images are labeled for each tag of interest: positive bags $\Omega$ versus negative bags $\bar{\Omega}$. For a given tag, its positive bags $\Omega$ consist of a set of multi-label images which are loosely related with the given tag, e.g., all these multi-label images are loosely tagged by the given tag and other inter-related tags and at least one of their image instances (i.e., image regions) has exact correspondence with the given tag. On the other hand, the negative bags $\bar{\Omega}$ consist of a set of multi-label images which are irrelevant with the given tag, e.g., the given tag is not used to tag all these multi-label images in the negative bags and none of their image instances (i.e., image regions) has exact correspondence with the given tag. One important issue for supporting multiple instance learning is how to identify the correspondences between multiple tags (given at the image level) and the image instances. 

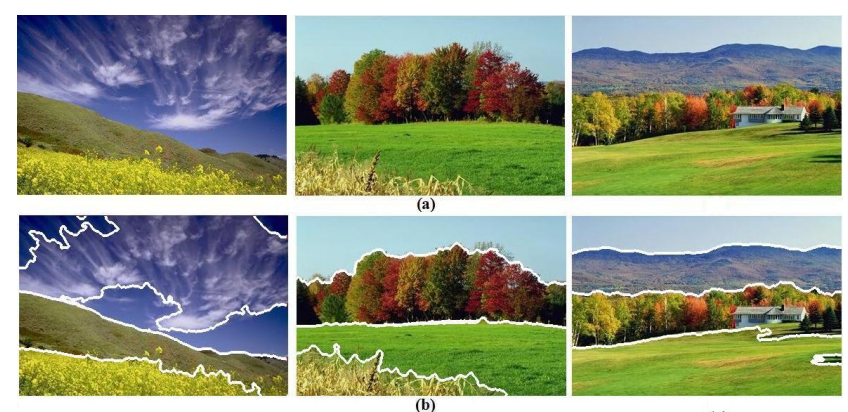

Figure 2: Multiple instances (image regions) for ambiguous image representation and feature extraction.

\subsection{Bags of Instances}

For automatic object detection and scene recognition applications, a good framework for image content representation that can capture more meaningful insights of the images may make the classifier training task easier to tackle. To leverage the loosely-tagged images for classifier training, having a good framework for ambiguous image representation is more important than having a strong learning algorithm. If a loosely-tagged image is treated as one single instance as shown in Fig. 2(a), the underlying ambiguous image semantics which correspond to multiple tags are mixed in the instance space, thus it could be very difficult to learn reliable classifiers by using such loosely-tagged images. On the other hand, if the loosely-tagged image is represented as a set of instances (image regions) as shown in Fig. 2(b), the mixed information (i.e., ambiguous image semantics) in the same image can be easier to be detached in the instance space, thus it can be less difficult to learn reliable classifiers by using such image instances.

As shown in Fig. 2(b), a new scheme is developed for ambiguous image representation by using bags of instances: (a) each loosely-tagged image is first partitioned into a set of image regions by using JSEG [4] and multiple segmentations are integrated to obtain more meaningful image regions (image instances) for object detection [5]; (b) each image region is treated as one instance; and (c) multi-modal visual features are extracted from each image instance to characterize its various visual properties more sufficiently.

By partitioning the loosely-tagged images into bags of instances, our ambiguous image representation framework can provide multiple advantages: (1) it can provide a good foundation to tackle the issue of loose tags by automatically identifying the correspondences between multiple tags (given at the image level) and the image instances (image regions); (2) it can provide a natural way to tackle the issue of semantics ambiguity (i.e., multiple tags) explicitly in the instance space, e.g., different image instances may relate to different tags; and (3) it is able to characterize the appearances of the objects effectively by integrating multiple segmentation results.

In our current implementations, we have extracted the following region-based visual features: 12-bins color histogram, top 3 dominant colors, 9-bins edge histogram, region shapes, region size and location of its center in an image, and Tamura textures. Each type of these visual features (i.e., one particular feature subset) is used to characterize one certain type of the visual properties of the image instances, and a suitable base kernel is designed for each type of the visual features for characterizing one certain type of the visual similarity contexts between the image instances. We skip the details for base kernel construction because it is beyond the scope of this paper and it can be found from our previous work [13].

For two image instances $u$ and $v$, their diverse visual similarity contexts are characterized more precisely by using a mixture of these base kernels (i.e., mixture-of-kernels) [13].

$$
\kappa(u, v)=\sum_{l=1}^{\tau} \alpha_{l} \kappa_{l}(u, v), \quad \sum_{l=1}^{\tau} \alpha_{l}=1
$$

where $\tau$ is the number of feature subsets (i.e., the number of base kernels), $\alpha_{l} \geq 0$ is the importance factor for the $l$ th base kernel $\kappa_{l}(u, v)$ and it can be obtained automatically.

For image annotation task, some pioneering work have been done on multiple instance learning (MIL) by treating each image as a bag of instances [7-10]. Because of the issue of multiple tags, our scenarios for multiple instance learning are significantly different: (a) each bag of instances (i.e., each multi-label image) is positive for multiple tags; and (b) multiple tags could be inter-related rather than independent, otherwise they may not be associated with the same image.

\subsection{Image Instance Clustering}

Because the object tags are loosely given at the image level, it is very important to develop new algorithms for determining the labels for the image instances (i.e., image regions) automatically. In order to identify the exact correspondences between multiple tags and the image instances, the image instances in the positive bags and the negative bags are first partitioned into multiple clusters according to their visual similarity contexts.

Our image instance clustering algorithm consists of the following steps: (a) The weights for all these $\tau$ feature subsets or base kernels are set equally, e.g., $\alpha_{1}=\alpha_{2}=\cdots$, = $\alpha_{\text {tau }}=\frac{1}{\tau}$. (b) The affinity propagation algorithm [6] is used to achieve more precise instance clustering. For the positive bags and the negative bags, a graph is first constructed to organize all the image instances according to their visual similarity contexts $\kappa(\cdot, \cdot)[2]$, where each node on the graph is one image instance and an edge between two nodes is used to characterize the visual similarity context between two image instances. By passing message between the nodes in the instance graph through affinity propagation, all these instances in the positive bags and the negative bags are independently grouped into multiple clusters according to their visual similarity contexts. (c) The optimal weights $\vec{\alpha}=\left[\hat{\alpha}_{1}\right.$, $\left.\cdots, \hat{\alpha}_{\tau}\right]$ for kernel combination are refined automatically by maximizing the inter-cluster separability and the intracluster compactness [2]:

$$
\underset{\alpha}{\max }\left\{\frac{1}{K} \sum_{l=1}^{K} \frac{\sigma\left(G_{l}\right)}{\mu\left(G_{l}\right)}\right\}
$$

subject to: $\quad \sum_{i=1}^{\tau} \alpha_{i}=1, \forall_{i}: \quad \alpha_{i} \geq 0$

We further define $X=\left[X_{1}, \cdots, X_{l}, \cdots, X_{k}\right]$ as the cluster indicators, and its component $X_{l}$ is a binary indicator for the appearance of the $l$ th cluster $G_{l}$,

$$
X_{l}(u)=\left\{\begin{array}{c}
1, \quad u \in G_{l} \\
0, \text { otherwise }
\end{array}\right.
$$

For a given instance cluster $G_{l}$, its inter-cluster separability $\mu\left(G_{l}\right)$ and its intra-cluster compactness $\sigma\left(G_{l}\right)$ are defined 
as:

$$
\begin{gathered}
\mu\left(G_{l}\right)=X_{l}^{t r}(D-W) X_{l}, \quad \sigma\left(G_{l}\right)=X_{l}^{t r} W X_{l} \\
W_{u, v}=\kappa(u, v), \quad D_{u, u}=\sum_{v=1}^{n} W_{u, v}
\end{gathered}
$$

The optimal kernel weights $\vec{\alpha}=\left[\hat{\alpha}_{1}, \cdots, \hat{\alpha}_{\tau}\right]$ are determined automatically by solving the following quadratic programming problem:

$$
\min _{\alpha}\left\{\frac{1}{2} \vec{\alpha}^{t r}\left(\sum_{l=1}^{K} \Omega\left(G_{l}\right) \Omega\left(G_{l}\right)^{t r}\right) \vec{\alpha}\right\}
$$

subject to: $\quad \sum_{i=1}^{\tau} \alpha_{i}=1, \quad \forall_{i}: \quad \alpha_{i} \geq 0$

$\Omega\left(G_{l}\right)$ is defined as:

$$
\begin{gathered}
\Omega\left(G_{l}\right)=\frac{\omega\left(G_{l}\right)}{\epsilon\left(G_{l}\right)-\omega\left(G_{l}\right)} \\
\omega\left(G_{l}\right)=\sum_{u \in G_{l}} \sum_{v \in G_{l}} \kappa_{i}(u, v), \quad \epsilon\left(G_{l}\right)=\sum_{v=1}^{n_{l}} \omega\left(G_{l}\right)
\end{gathered}
$$

(d) These steps are performed repeatedly until convergence.

The instance clusters in the positive bags can further be partitioned into two groups: relevant instance clusters (which are strongly related with the given tag and the semantics of their instances can be interpreted precisely by the given tag) versus irrelevant instance clusters. Obviously, the ratio between the irrelevant instance clusters and the relevant instance clusters could be arbitrarily high.

\subsection{Relevant Cluster Identification}

When large amounts of positive bags are available, the relevant clusters may have larger sizes because the relevant instances may share some common visual properties for the given object class (i.e., the given tag) and they can be assigned into the same cluster. The irrelevant clusters may have smaller sizes because the irrelevant image instances may belong to many different object classes and they may have less correlations on their visual properties. On the other hand, the image instances in the negative bags can also be partitioned into a set of irrelevant clusters.

For a given instance cluster $G_{i}$ (it could be either relevant cluster or irrelevant cluster) in the positive bags $\Omega$ and a given irrelevant cluster $G_{j}$ in the negative bags $\bar{\Omega}$, their intercluster visual similarity context is defined as:

$$
\delta\left(G_{i}, G_{j}\right)=\frac{1}{2\left|G_{i}\right|\left|G_{j}\right|} \sum_{u \in G_{i}} \sum_{v \in G_{j}}[\hat{\kappa}(u, v)+\bar{\kappa}(u, v)]
$$

where $\left|G_{i}\right|$ and $\left|G_{j}\right|$ are the total numbers of the image instances for the clusters $G_{i}$ and $G_{j}, \hat{\kappa}(u, v)$ is the pairwise visual similarity context between the image instance $u$ from $G_{i}$ and the image instance $v$ from $G_{j}$ by using the kernel weights for the instance cluster $G_{i}$, and $\bar{\kappa}(u, v)$ is the pairwise visual similarity context between two image instances $u$ and $v$ by using the kernel weights for the instance cluster $G_{j}$. All these kernel weights are automatically provided by the instance clustering process.

The best-matched cluster pair $\left(G_{i}, G_{k}\right)$ between the positive bags and the negative bags is determined by:

$$
\bar{\delta}\left(G_{i}, G_{k}\right)=\max \left\{\delta\left(G_{i}, G_{j}\right) \mid G_{i} \in \Omega, G_{j} \in \bar{\Omega}\right\}
$$

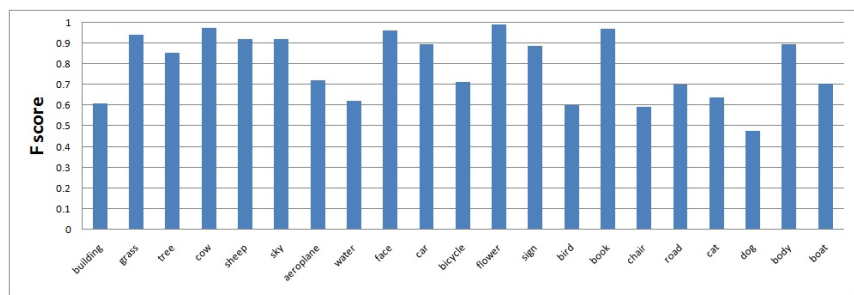

Figure 3: The F scores for our multiple instance learning (instance label identification) algorithm on MSRC image set (using ground truth segmentation).

This process for best-matched cluster pair determination is continued until all these instance clusters (they could be either relevant clusters or irrelevant clusters) in the positive bags have found the best-matched irrelevant cluster in the negative bags.

The instance clusters in the positive bags are then partitioned into two groups according to their pairwise intercluster visual similarity contexts with the irrelevant clusters in the negative bags: positive groups versus negative groups. The relevant clusters in the positive bags should be far away from the irrelevant clusters in the negative bags (i.e., with small values of the inter-cluster similarity contexts $\bar{\delta}(\cdot, \cdot)$ ) [9], they are assigned into the positive groups. On the other hand, the irrelevant clusters in the positive bags may be close to the irrelevant clusters in the negative bags (i.e., with large values of the inter-cluster similarity contexts $\bar{\delta}(\cdot, \cdot))$, they can be assigned into the negative groups.

It is worth noting that some irrelevant clusters in the positive bags may not have strong correlations with the irrelevant clusters in the negative bags (i.e., they may belong to different object classes), thus such irrelevant clusters in the positive bags may first be assigned into the positive groups. Compared with the relevant clusters in the positive groups, such the irrelevant clusters (which are first assigned into the positive groups) may have smaller sizes, thus they can further be separated from the relevant clusters according to their size differences. For the relevant clusters in the positive bags, there is a significant difference with the irrelevant clusters in the positive bags either on their correlations with the irrelevant clusters in the negative bags or on their sizes, thus it is easy for us to separate the relevant clusters from the irrelevant clusters in the positive bags. When the relevant clusters are identified from the irrelevant clusters in the positive bags, the given tag is treated as the ground-truth label for all their image instances in the relevant clusters.

Through analyzing both the cluster sizes and the values of their inter-cluster correlations, we can separate the relevant clusters from the irrelevant clusters more precisely and identify the exact correspondences between the image instances and multiple tags more accurately. As shown in Fig. 3, the traditional F score (which is defined as the harmonic mean of precision and recall) is used to evaluate the performance of our multiple instance learning algorithm for instance label identification. The F score for performance evaluation is defined as:

$$
F=\frac{2 \times \text { precision } \times \text { recall }}{\text { precision }+ \text { recall }}
$$

where precision and recall are the well-known precision and recall rates in the literature.

The image instances in the relevant clusters, which their semantics have better correspondence with the given tag, 
Table 1: Inter-object correlations.

\begin{tabular}{|c|c|c|c|c|c|c|c|}
\hline object pair & $\phi$ & object pair & $\phi$ & object pair & $\phi$ & object pair & $\phi$ \\
\hline grass-building & 0.04 & horse-cow & 0.31 & cat-dog & 0.85 & car-bicycle & 0.53 \\
bird-aeroplane & 0.54 & car-aeroplane & 0.55 & boat-grass & 0.02 & dog-cow & 0.51 \\
sign-building & 0.63 & sheep-horse & 0.66 & body-dog & 0.83 & body-cat & 0.79 \\
road-building & 0.72 & road-sign & 0.59 & boat-aeroplane & 0.73 & mountain-road & 0.62 \\
cat-grass & 0.03 & tree-cat & 0.72 & book-grass & 0.06 & sky-book & 0.02 \\
boat-sky & 0.06 & aeroplane-mountain & 0.43 & mountain-water & 0.75 & body-sky & 0.06 \\
\hline
\end{tabular}

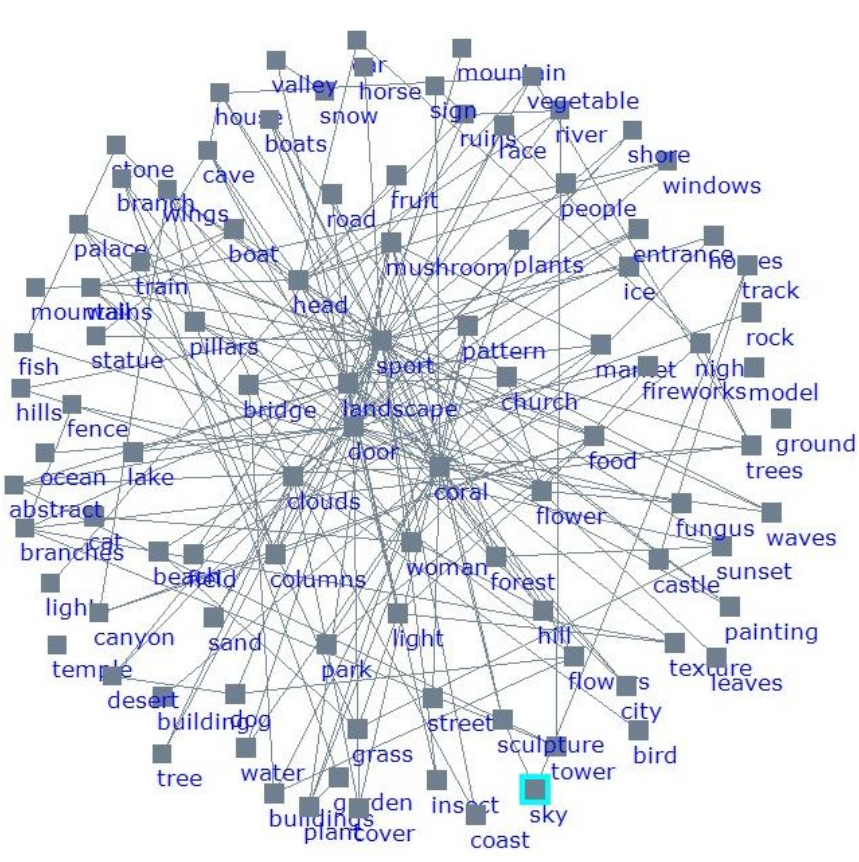

Figure 4: Part of our object correlation network.

can be selected as the relevant image instances and be used to train the object classifier for the given object class (i.e., the given tag). Thus our multiple instance learning algorithm for instance label identification can allow us to address the issue of loose tags effectively, which can provide a good environment to leverage the loosely-tagged images for object classifier training.

\section{OBJECT CORRELATION NETWORK}

An object correlation network is constructed to characterize the inter-object correlations more precisely and provide a good environment to determine the inter-related learning tasks directly in the feature space. Our object correlation network consists of two key components: object classes and their inter-object correlations.

For two given object classes $C_{i}$ and $C_{j}$, their inter-object visual similarity context $\gamma\left(C_{i}, C_{j}\right)$ is determined by:

$$
\gamma\left(C_{i}, C_{j}\right)=\frac{1}{2\left|C_{i}\right|\left|C_{j}\right|} \sum_{u \in C_{i}} \sum_{v \in C_{j}}[\hat{\kappa}(u, v)+\bar{\kappa}(u, v)]
$$

where $\left|C_{i}\right|$ and $\left|C_{j}\right|$ are the total numbers of the image instances for the object classes $C_{i}$ and $C_{j}, \hat{\kappa}(u, v)$ is the kernelbased similarity context between two image instances $u$ and $v$ by using the kernel weights for the object class $C_{i}$, and $\bar{\kappa}(u, v)$ is the kernel-based similarity context between two image instances $u$ and $v$ by using the kernel weights for the object class $C_{j}$. All these kernel weights are automatically provided by the instance clustering process.

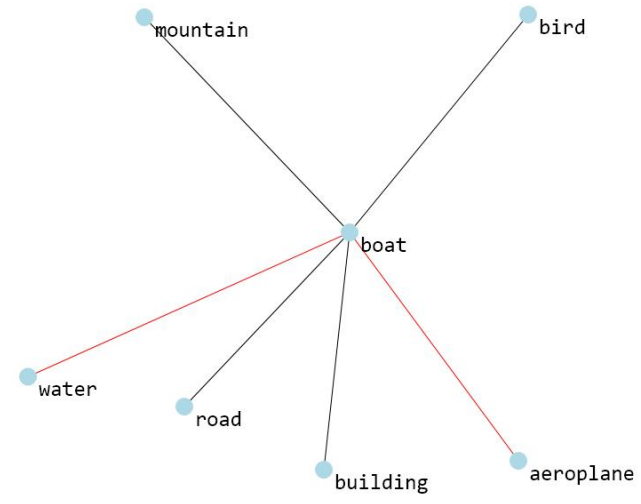

Figure 5: The inter-related object classes for "boat".

The co-occurrence correlation $\rho\left(C_{i}, C_{j}\right)$ between two object classes $C_{i}$ and $C_{j}$ is defined as:

$$
\rho\left(C_{i}, C_{j}\right)=-P\left(C_{i}, C_{j}\right) \log \frac{P\left(C_{i}, C_{j}\right)}{P\left(C_{i}\right)+P\left(C_{j}\right)}
$$

where $P\left(C_{i}, C_{j}\right)$ is the co-occurrence probability for two object classes $C_{i}$ and $C_{j}$ in our image collections, $P\left(C_{i}\right)$ and $P\left(C_{j}\right)$ are the occurrence probabilities for $C_{i}$ and $C_{j}$.

For two given object classes $C_{i}$ and $C_{j}$, their visual similarity context $\gamma\left(C_{i}, C_{j}\right)$ and their co-occurrence correlation $\rho\left(C_{i}, C_{j}\right)$ are first normalized into the same interval. The inter-object correlation $\phi\left(C_{i}, C_{j}\right)$ between two object classes $C_{i}$ and $C_{j}$ is finally defined as:

$$
\phi\left(C_{i}, C_{j}\right)=\eta \cdot \bar{\gamma}\left(C_{i}, C_{j}\right)+(1-\eta) \cdot \bar{\rho}\left(C_{i}, C_{j}\right)
$$

where $\eta$ is the weighting factor and it is determined through cross-validation, $\bar{\gamma}\left(C_{i}, C_{j}\right)$ and $\bar{\rho}\left(C_{i}, C_{j}\right)$ are the normalized visual similarity context and co-occurrence correlation. The weighting factor is set as $\eta=0.6$ in our current implementation because the visual similarity contexts are more important than the co-occurrence correlations for inter-object correlation characterization.

Some experimental results on the inter-object correlations $\phi(\cdot, \cdot)$ are given in Table 1 . Part of our object correlation network for our image sets is shown in Fig. 4, where each object class is linked with multiple most relevant object classes with larger values of $\phi(\cdot, \cdot)$. One can find that our object correlation network can characterize the correlations among the object classes effectively. Our object correlation network may have multiple advantages: (a) It can characterize the inter-object correlations explicitly and provide a good environment to identify the inter-related learning tasks directly in the feature space. (b) It can provide a good environment to integrate the training instances from multiple interrelated object classes for training their classifiers jointly and bring more powerful inference schemes to enhance their discrimination and adaptation power significantly. 


\section{STRUCTURED LEARNING}

The object classes are inter-related and such inter-object correlations can be represented explicitly by the object correlation network and can be represented precisely by the strengths of their inter-object correlations $\phi(\cdot, \cdot)$. When a large number of object classes come into view, directly modeling of such inter-object correlations over the whole object correlation network becomes computationally intractable. In this paper, a multi-task structured SVM scheme is developed by incorporating the first-order nearest neighbors (i.e., clique for each object class on the object correlation network), multi-task learning and structured SVM to leverage the inter-object correlations to achieve more accurate training of a large number of inter-related object classifiers.

For a given object class (one example is shown in Fig. 5 ), its first-order nearest neighbors on the object correlation network are strongly correlated and their training instances may share similar visual properties. Thus isolating these inter-related object classes and training their classifiers independently are not appropriate. In order to leverage the inter-object correlations for training the inter-related object classifiers jointly, it is very important to develop new algorithms for integrating multi-task learning with structured SVM to model the inter-task relatedness more precisely. The idea behind multi-task learning is that if multiple interrelated learning tasks share a common prediction component, such common prediction component can be estimated more accurately by considering these inter-related learning tasks together [13-14]. When multiple tags are jointly used for image tagging, the corresponding object classes should be inter-related rather than independent and thus learning the classifiers for these inter-related object classes should be considered jointly. Our object correlation network can provide a good environment for identifying such inter-related learning tasks directly in the feature space. The idea behind structured SVM [23-24] is to exploit the inter-label correlations in the label space for supporting structure prediction.

In this paper, a multi-task structured SVM scheme is developed by incorporating the object correlation network, multi-task learning and structured SVM to enhance the discrimination power of a large number of inter-related object classifiers: (a) The object correlation network is used to identify the inter-related learning tasks directly in the feature space, e.g., training multiple inter-related object classifiers jointly; (b) The inter-task relatedness is characterized explicitly by using the strengths of the inter-object correlations $\phi(\cdot, \cdot)$ and a common prediction component is used to model the inter-task relatedness and shared among these inter-related object classifiers; (c) The structured SVM is integrated with multi-task learning to model the inter-task relatedness more precisely and estimate the common prediction component more accurately. By seamlessly integrating multi-task learning with structured SVM, our multi-task structured SVM algorithm is able to exploit the inter-object correlations explicitly in the input space (i.e., the feature space for classifier training and testing), thus it can provide a new approach for inter-related classifier training and address the issue of multiple tags more effectively.

In our multi-task structured SVM scheme, a common regularization term $W_{0}$ of the SVM classifier is used to model the inter-task relatedness among multiple SVM classifiers for the inter-related object classes. For one given object class
$C_{j}$, its classifier is defined as:

$$
f_{C_{j}}(x)=\sum_{C_{t} \in \mathcal{T}} \gamma_{t}\left(W_{0}+V_{t}\right)^{t r} \Phi_{t}(x)
$$

where $W_{0}$ is the common regularization term shared among the classifiers for multiple inter-related object classes centered by $C_{j}$ as shown in Fig. $5, V_{t}$ is the individual regularization term for the classifier between the given object class $C_{j}$ and its neighbor $C_{t}, \gamma_{t}$ is the weight how class $C_{t}$ contributes the classification of $C_{j}$.

The common regularization term $W_{0}$ is used to model the inter-task relatedness and shared among the classifiers for multiple inter-related object classes. $W_{0}$ can be estimated more reliably by minimizing their joint objective function $J$ for $T$ inter-related learning tasks.

$$
J=\frac{1}{2}\left(\left\|W_{0}\right\|^{2}+\sum_{t=1}^{T} \lambda_{t}\left\|V_{t}\right\|^{2}\right)+c_{0} \sum_{t=1}^{T} \sum_{i=1}^{n_{j}} \xi_{t i}+\sum_{t=1}^{T} c_{t} \sum_{i=1}^{n_{t}} \eta_{t i}
$$

where $\xi_{t i} \geq 0, \eta_{t i} \geq 0, n_{j}$ and $n_{t}$ are the total number of training instances for the object classes $C_{j}$ and $C_{t}$.

To solve the joint optimization problem, we use the Lagrangian Principle. We add a dual set of variables, one for each constraint and get the Lagrangian $L$ of the optimization problem:

$$
\begin{aligned}
L=J & -\sum_{t=1}^{T} \sum_{i}^{n_{j}} \beta_{t i}\left(\left\langle W_{0}+V_{t}, \Phi_{t}\left(x_{j i}\right)\right\rangle+\xi_{t i}-1\right) \\
& +\sum_{t=1}^{T} \sum_{i=1}^{n_{t}} \overline{\beta_{t i}}\left(\left\langle W_{0}+V_{t}, \Phi_{t}\left(x_{t i}\right)\right\rangle-\eta_{t i}+1\right) \\
& -\sum_{t=1}^{T} \sum_{i=1}^{n_{j}} \sigma_{t i} \xi_{t i}-\sum_{t=1}^{T} \sum_{i=1}^{n_{t}} \overline{\sigma_{t i}} \eta_{t i}
\end{aligned}
$$

We now seek a saddle point of the Lagrangian $L$, e.g., the partial difference of $L$ satisfied:

$$
\begin{aligned}
\frac{\partial L}{\partial W_{0}} & =W_{0}-\sum_{t=1}^{T} \sum_{i=1}^{n_{j}} \beta_{t i} \Phi_{t}\left(x_{j i}\right)+\sum_{t=1}^{T} \sum_{i=1}^{n_{t}} \overline{\beta_{t i}} \Phi_{t}\left(x_{t i}\right) \\
\frac{\partial L}{\partial V_{t}} & =\lambda_{t} V_{t}-\sum_{i=1}^{n_{j}} \beta_{t i} \Phi_{t}\left(x_{j i}\right)+\sum_{i=1}^{n_{t}} \overline{\beta_{t i}} \Phi_{t}\left(x_{t i}\right) \\
\frac{\partial L}{\partial \xi_{t i}} & =c_{0}-\beta_{t i}-\sigma_{t i}, \quad \frac{\partial L}{\partial \eta_{t i}}=c_{t}-\overline{\beta_{t i}}-\overline{\sigma_{t i}}
\end{aligned}
$$

and we can get:

$$
\begin{aligned}
W_{0} & =\sum_{t=1}^{T} \sum_{i=1}^{n_{j}} \beta_{t i} \Phi_{t}\left(x_{j i}\right)-\sum_{t=1}^{T} \sum_{i=1}^{n_{t}} \overline{\beta_{t i}} \Phi_{t}\left(x_{t i}\right) \\
V_{t} & =\frac{1}{\lambda_{t}}\left(\sum_{i=1}^{n_{j}} \beta_{t i} \Phi_{t}\left(x_{j i}\right)-\sum_{i=1}^{n_{t}} \overline{\beta_{t i}} \Phi_{t}\left(x_{t i}\right)\right) \\
c_{0} & =\beta_{t i}+\sigma_{t i}, \quad c_{t}=\overline{\beta_{t i}}+\overline{\sigma_{t i}}
\end{aligned}
$$

The dual form of the problem is then simplified as:

$L=\sum_{t=1}^{T} \sum_{i=1}^{n_{j}} \beta_{t i}+\sum_{t=1}^{T} \sum_{i=1}^{n_{t}} \overline{\beta_{t i}}-\frac{1}{2}\left(\left\|W_{0}\right\|^{2}+\sum_{t=1}^{T} \lambda_{t}\left\|V_{t}\right\|^{2}\right)$ 

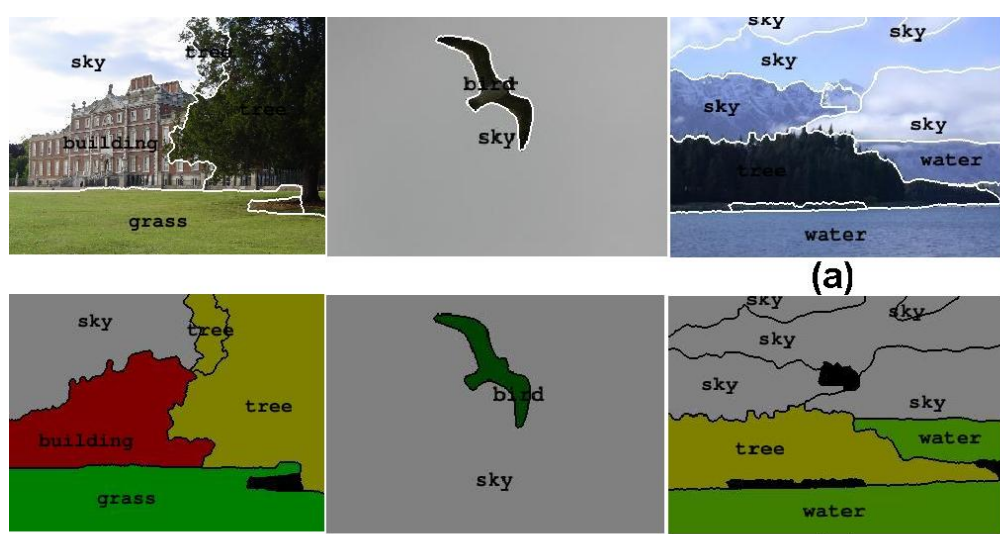

(a)

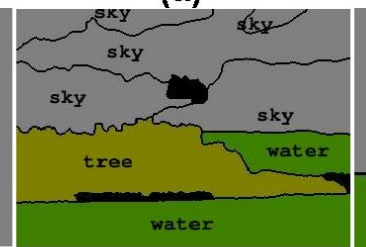

(b)
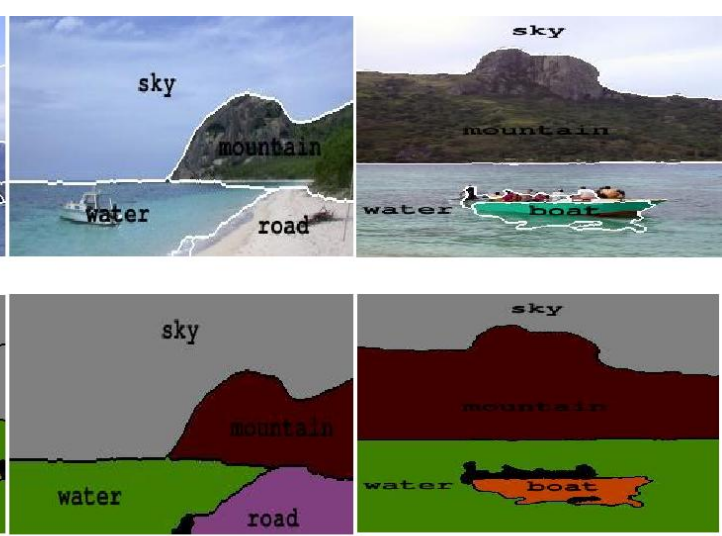

Figure 6: Object detection results by our multi-task structured SVM algorithm.

Given the training image instances for $T$ inter-related object classes on the object correlation network, the margin maximization process for object classifier training is then transformed into a quadratic problem:

$$
\begin{aligned}
\max _{\beta_{t i}, \beta_{t i}} L & =\sum_{t=1}^{T} \sum_{i=1}^{n_{j}} \beta_{t i}+\sum_{t=1}^{T} \sum_{i=1}^{n_{t}} \overline{\beta_{t i}} \\
& -\frac{1}{2}\left[\sum_{t, s=1}^{T} \sum_{i=1}^{n_{j}} \sum_{l=1}^{n_{l}} \beta_{t i} \beta_{s l} K_{t s}\left(x_{j i}, x_{j l}\right)\right. \\
& -\sum_{t, s=1}^{T} \sum_{i=1}^{n_{j}} \sum_{l=1}^{n_{s}} \beta_{t i} \overline{\beta_{s l}} K_{t s}\left(x_{j i}, x_{s l}\right) \\
& -\sum_{t, s=1}^{T} \sum_{i=1}^{n_{t}} \sum_{k=1}^{n_{j}} \overline{\beta_{t i}} \beta_{s k} K_{t s}\left(x_{t i}, x_{k j}\right) \\
& +\sum_{t, s=1}^{T} \sum_{i=1}^{n_{t}} \sum_{k=1}^{n_{s}} \overline{\beta_{t i} \beta_{s k}} K_{t s}\left(x_{t i}, x_{s k}\right) \\
& +\sum_{t=1}^{T} \frac{1}{\lambda_{t}}\left(\sum_{i, l=1}^{n_{j}} \beta_{t i} \beta_{t l} K_{t}\left(x_{j i}, x_{j l}\right)\right. \\
& -2 \sum_{i=1}^{n_{j}} \sum_{l=1}^{n_{t}} \beta_{t i} \overline{\beta_{t l}} K_{t}\left(x_{j i}, x_{j l}\right) \\
& \left.\left.+\sum_{i, l=1}^{n_{t}} \overline{\beta_{t i} \beta_{t l}} K_{t}\left(x_{t i}, x_{t l}\right)\right)\right]
\end{aligned}
$$

subject to: $\quad \forall_{i}: \quad \forall_{t}: \quad \beta_{t i} \geq 0, \quad \overline{\beta_{t i}} \geq 0$

To deal with the structured prediction problem, it is very attractive to construct a joint kernel function that is better suited to joint-space support estimation. In this paper, a tensor products is incorporated to define the joint kernel $\kappa\left(\left(x_{i}, y_{i}\right),\left(x_{j}, y_{j}\right)\right)$ as:

$$
\kappa\left(\left(x_{i}, y_{i}\right),\left(x_{j}, y_{j}\right)\right)=\kappa\left(x_{i}, x_{j}\right) \kappa_{s}\left(y_{i}, y_{j}\right)
$$

where $\kappa\left(x_{i}, x_{j}\right)$ is the mixture-of-kernels for the visual features as defined in Eq. (1) and $\kappa_{s}\left(y_{i}, y_{j}\right)$ is the semantic kernel to characterize the semantic similarity context between two object classes and their labels $y_{i}$ and $y_{j}$ (i.e., inter-object correlation on the label space).

By learning from a joint training image set $\Omega=\left\{\left(x_{i t}, y_{i t}\right) \mid i=\right.$ $1, \cdots, n ; t=1, \cdots, T\}$ for $T$ inter-related object classes on the object correlation network, the classifier for the given object class $C_{j}$ can be determined as:

$$
\begin{gathered}
f_{C_{j}}(x)=\sum_{l=1}^{\tau} \alpha_{l} \sum_{h, t=1}^{T} \gamma_{t} \kappa_{s}(t, h)\left(\sum_{i=1}^{n_{j}} \beta_{h i} \kappa_{l}\left(x_{j i}, x\right)-\sum_{i=1}^{n_{h}} \overline{\beta_{h i}} \kappa_{l}\left(x_{h i}, x\right)\right) \\
+\sum_{l=1}^{\tau} \sum_{t=1}^{T} \frac{\alpha_{l} \gamma_{t}}{\lambda_{t}}\left(\sum_{i=1}^{n_{j}} \beta_{t i} \kappa_{l}\left(x_{j i}, x\right)-\sum_{i=1}^{n_{t}} \overline{\beta_{t i}} \kappa_{l}\left(x_{t i}, x\right)\right)
\end{gathered}
$$

One can observe that our classifiers for inter-related object classes consist of two components: (a) individual prediction component; and (b) common prediction component.

By learning two different sets of the kernel coefficients $\alpha$ and $\bar{\alpha}$ simultaneously, our multi-task structured SVM algorithm can automatically determine two separable feature subsets, which can effectively characterize both the common visual properties shared among all these $T$ inter-related object classes and the individual visual properties for each particular object class. The feature subsets and their base kernels, which are used to construct the common prediction component for multiple inter-related object classifiers (i.e., with larger values of $\bar{\alpha}$ ), are less important for the individual prediction components for these inter-related object classifiers (i.e., with smaller values or even zero values of $\alpha$ ).

By learning two different sets of the weights $\beta$ and $\bar{\beta}$ for the training instances simultaneously, our multi-task structured SVM algorithm can automatically establish two independent decision boundaries for both the common prediction component (shared among the inter-related discriminant functions) and the individual prediction component of the discriminant function for each particular object class. The training instances, which are used to construct the common prediction component for multiple inter-related object classifiers (i.e., support vectors with large values of $\bar{\beta}$ ), are less important for the individual prediction components for these inter-related object classifiers (i.e., with smaller values or even zero values of weights $\beta$ ).

The kernel coefficients $\bar{\alpha}$ and the weights $\bar{\beta}$ are fixed for all these $T$ inter-related discriminant functions to characterize their common prediction component. By integrating the training instances for multiple inter-related object classes to learn a common prediction component and separating it from their individual prediction components, our multitask structured SVM algorithm can significantly enhance the discrimination power and the generalization ability of the inter-related object classifiers. 


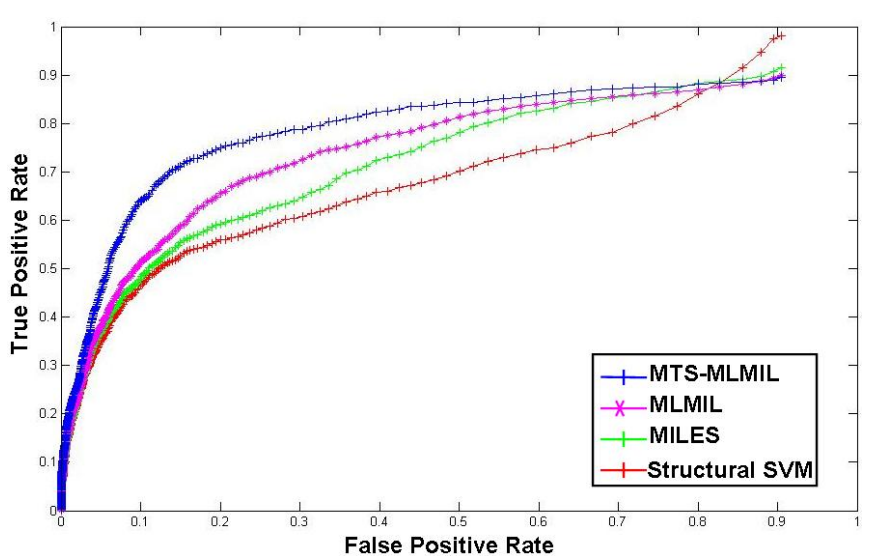

Figure 7: ROC curves for performance comparison: our multi-task structured SVM (MTS-MLMIL) algorithm versus other most relevant algorithms.

The inter-related object classifiers for a set of object classes are trained in our current implementation. After the object classifiers are trained, they are further used to identify the objects from the test images and recommend the suitable tags for automatic image annotation. Our automatic object detection and tag recommendation algorithm takes the following key steps: (a) The test image is first segmented into multiple image regions. (b) Each image region (image instance) is then classified into the most relevant object class with the maximum value of the confidence (posterior probability). (c) The tags for describing the semantics of these object classes are then selected for annotating the given test image automatically. Some experimental results on object detection (tag recommendation) are given in Fig. 6. One can observe that our multi-task structured SVM scheme can directly learn the object detectors from the loosely-tagged images and precisely localize the objects in the images.

Most existing algorithms for object detection are often handled by combining multiple binary classifiers, thus they may have square complexity with the number of object classes $N$, i.e., $O\left(N^{2}\right)$. On the other hand, our algorithm for object detection can achieve linear complexity with the size of the object correlation network $N$, i.e., $O(N)$, thus it is very attractive for dealing with a large number of object classes.

\section{ALGORITHM EVALUATION}

Our experiments are performed on two sets of looselytagged images: (a) 3, 814 MSRC image instances (image regions) [3] and 30K Corel images [26]; (b) 1.0 million looselytagged images which are collected from Flickr [1-2]. Because MSRC images and Corel images are easy to obtain, we use them as our test image sets, so that other researchers can easily assess the real performances of our algorithms. On the other hand, Flickr can allow us to collect large-scale and realistic loosely-tagged images, it is very attractive to use such realistic loosely-tagged images for developing new algorithms which can tackle the issue of tag uncertainty and learn the object classifiers reliably. Thus Flickr image set is used as the training image set in our experiments [1-2].

To assess the effectiveness of our proposed algorithms, our algorithm evaluation work focuses on comparing the performance differences between various approaches for object classifier training: (a) our multi-task structured SVM (MTSMLMIL) algorithm versus structured SVM algorithm (ex- ploiting the inter-label correlations in the output space) [2324]; (b) our multi-task structured SVM (MTS-MLMIL) algorithm versus MILES (without exploiting the inter-label correlations explicitly) [10], and (c) our multi-task structured SVM (MTS-MLMIL) algorithm versus multi-label MIL (MLMIL) technique developed by Zha et al. [22]. In this paper, AUC (area under ROC curve) is adopted to evaluate the classification performance [25], which describes the probability that a randomly chosen positive image will be ranked higher than a randomly chosen negative image.

By using the same set of multi-modal visual features for image content representation, we have compared the performance differences between two approaches to integrate loosely-tagged images for object classifier training: MILES approach [10] versus our multi-task structured SVM (MTSMLMIL) algorithm. As shown in Fig. 7 and Fig. 8, one can observe that our multi-task structured SVM (MTS-MLMIL) algorithm can significantly improve the accuracy for detecting the inter-related object classes. The significant improvement on the detection accuracy benefits from two components: (1) The object classifiers for the inter-related object classes are trained jointly by leveraging their inter-object correlations for object classifier training, thus our multitask structured SVM algorithm can address the issue of multiple tags more effectively. Our multi-task structured SVM algorithm can address the issue of visual ambiguity more effectively by learning the inter-related classifiers for the inter-related object classes jointly. It can also enhance the discrimination and adaptation power of the inter-related object classifiers significantly by learning from the training instances for other inter-related object classes on the object correlation network. Incorporating the training instances from other inter-related object classes for classifier training will significantly enhance the generalization ability of their classifiers, especially when the available training instances for the given object class may not be representative for large amounts of unseen test images. On the other hand, MILES does not consider the inter-object (inter-label) correlations explicitly, which may result in lower accuracy rates for detecting some inter-related object classes. (2) Through an instance clustering and inter-cluster correlation analysis process, our multi-task structured SVM algorithm can address the issue of loose tags more effectively, which is crucial to leverage the loosely-tagged images for object classifier training.

Two existing approaches have considered the inter-label (inter-object) correlations for classifier training: (a) structured SVM algorithm [23-24]; and (b) multi-label multiple instance learning (MLMIL) algorithm [22]. Our multi-task structured SVM (MTS-MLMIL) algorithm is somewhat similar in spirit with these two existing approaches for object classifier training, but it significantly different from them in multiple important aspects. Compared with both the structured SVM algorithm and the multi-label multiple instance learning algorithm, our multi-task structured SVM algorithm can have multiple advantages: (a) it can explicitly model the inter-object correlations and the inter-task relatedness in the inter-related object classifiers (i.e., common regularization component $W_{0}$ ), which may provide a good environment to leverage the inter-object correlations and the inter-task relatedness for inter-related classifier training and enhance their discrimination power significantly; (b) it can save the cost for object detection by exploiting the inter- 


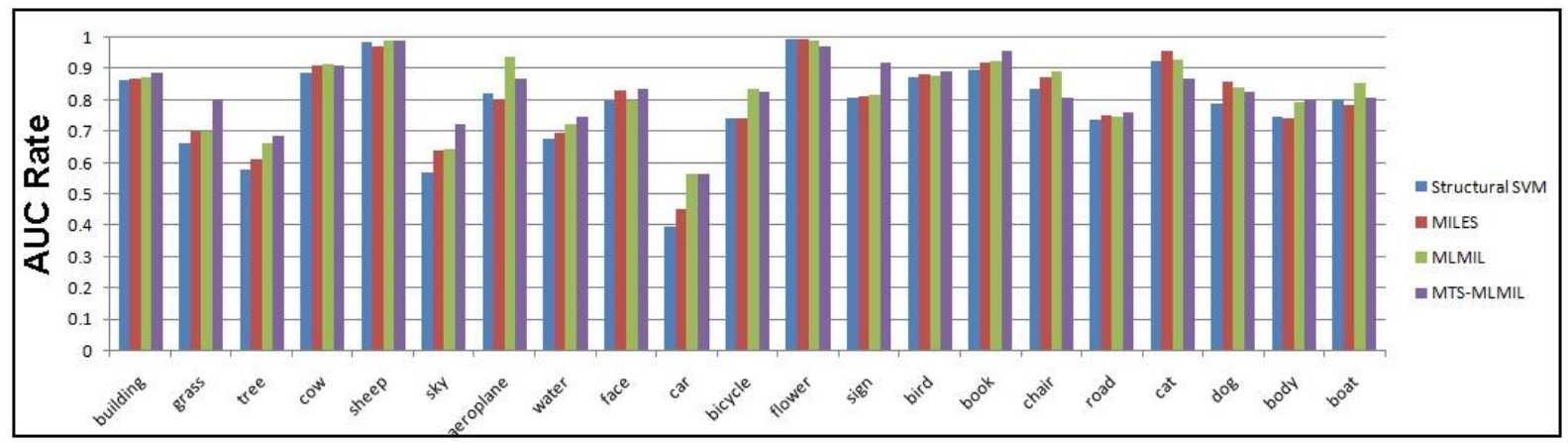

Figure 8: Performance comparison on AUC rates: our multi-task structured SVM (MTS-MLMIL) algorithm versus other most relevant algorithms.

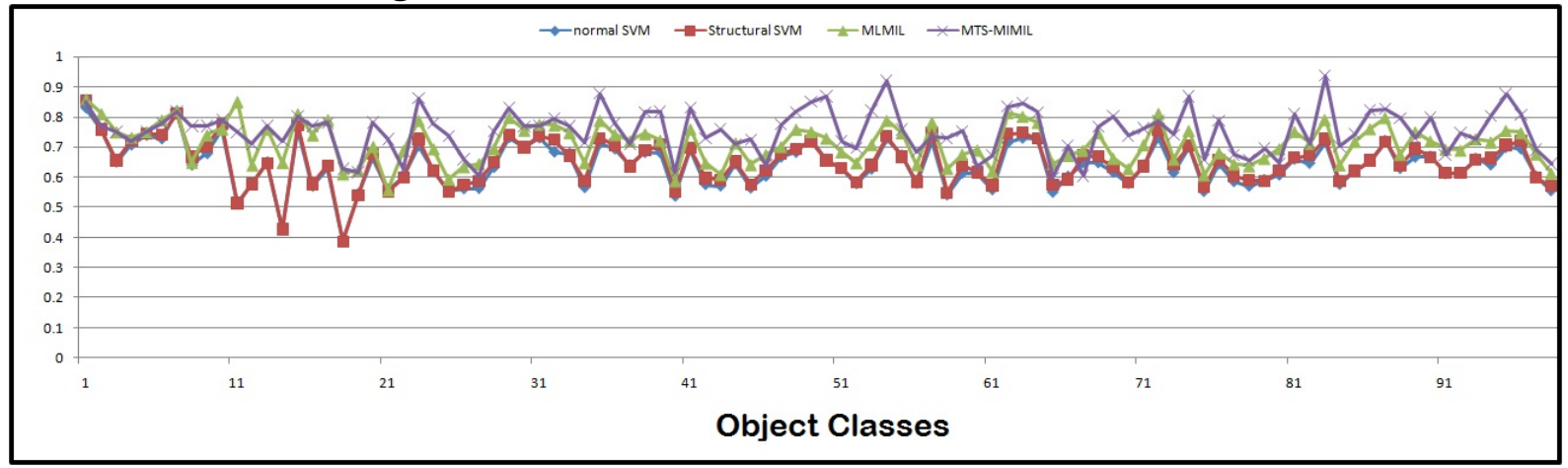

Figure 9: Performance comparison on 30K Corel images with 98 object classes.

Table 2: Average AUC scores on MSRC [3].

\begin{tabular}{|c|c|}
\hline algorithms & average AUC scores \\
\hline Structural SVM [22-23] & 0.6952 \\
MILES [10] & 0.7304 \\
MLMIL [22] & 0.7539 \\
Our MTS-MLMIL & 0.7965 \\
\hline
\end{tabular}

object correlations in the feature space. On the other hand, both the structured SVM algorithm and the multi-label multiple instance learning algorithm model the inter-object correlations in the output space (label space) rather than in the input space (feature space). In average, our multi-task structured SVM algorithm has better performance than the structured SVM algorithm and the multi-label multiple instance learning algorithm as shown in Table 2.

By generalizing multi-class SVM algorithm, the structured SVM algorithm focuses on supporting structural output prediction for a large number of SVM object classifiers, e.g., modeling the inter-object contexts in the output space and exploiting the inter-object contexts at the label space rather than in the feature space. On the other hand, our multitask structured SVM (MTS-MLMIL) algorithm can directly model the inter-task relatedness in the feature space (i.e., the input space for classifier training and testing) and explicitly exploit the inter-object correlations to achieve more effective training of a large number of inter-related object classifiers. As shown in Fig. 7 and Fig. 8, our multi-task structured SVM algorithm can have better performance (higher AUC rates) as compared with the structured SVM algorithm.

We have also compared the performances between our multi-task structured SVM (MTS-MLMIL) algorithm versus multi-label MIL (MLMIL) technique developed by Zha et al. [22]. By explicitly modeling the inter-task relatedness in the feature space rather than in the label space, our multi-task structured SVM algorithm can provide a good environment to leverage the inter-object correlations and the inter-task relatedness for inter-related object classifier training, which may result in higher discrimination powers for a large number of inter-related object classifiers. Compared with the multi-label multiple instance learning algorithm, our multi-task structured SVM algorithm can achieve very competitive performance as shown in Fig. 7 and Fig. 8. For some object classes, the MLMIL algorithm can obtain a little bit higher accuracy rates, but our multi-task structured SVM algorithm can in average achieve better accuracy rate as shown in Table 2 for MSRC image set with 21 object classes [3, 22].

Besides MSRC images, we have also compared the performances between our proposed algorithm (MTS-MLMIL) versus normal SVM and multi-label MIL(MLMIL) on 30K Corel images for 98 concept categories [26], where the original images are associated with totally 5k tags and 98 frequent object tags are chosen to be our distinguished concept categories. In average, each Corel image may have more than 10 image regions (instances), thus there are totally $300 \mathrm{~K}$ instances which are too large to be handled effectively by using one single PC (i.e., pre-compute and store the kernel-based similarity matrix for all these $300 \mathrm{~K}$ instances). When the size of image instances reaches one million, some existing techniques for kernel-based image clustering and SVM classifier training (such as LIBSVM [28]) may take years to run out a $O\left(\mathrm{~m}^{3}\right)$ algorithm on a single PC. Thus it is very attractive to develop a distributed computing framework to enable kernel-based image clustering and SVM classifier training.

To address the issue of computational complexity more effectively, two approaches are used: (a) only the first-order neighbors and their image instances are integrated for inter- 
Table 3: Average AUC scores on Corel images.

\begin{tabular}{|c|c|}
\hline algorithms & average AUC scores \\
\hline normal SVM [28] & 0.6438 \\
Structural SVM [22-23] & 0.6512 \\
MLMIL [22] & 0.7236 \\
Our MTS-MLMIL & 0.7549 \\
\hline
\end{tabular}

related classifier training; and (b) cascade learning framework is incorporated for training the SVM classifiers in a distributed way [27]. The idea of the cascade learning framework is to equally divide the image instances and iteratively aggregate the final SVM classifiers. For a given object class on the object correlation network, all the positive image instances for itself and its first-order neighbors are integrated for joint classifier training. To enhance the discrimination power of a large number of inter-related object classifiers, the cascade framework is used to sample the positive instances from other object classes which are not the first-order neighbors for the given object class.

The original version of the MILES algorithm focuses on solving a 1-norm SVM problem over all the training instances, it is too time-consuming to leverage the MILES algorithm for training the classifiers for a large number of inter-related object classes. On the other hand, it is not an easy task to adapt the MILES algorithm into the cascade learning framework, thus it is very hard for us to obtain the performance of the MILES algorithm on a large number of inter-related object classes by using a large Corel image set for classifier training. Based on this observation, we do not compare the performance difference between our multitask structured SVM algorithm and the MILES algorithm on $30 \mathrm{~K}$ Corel image set with 98 object classes. As shown in Fig. 9 and Table 3, our proposed algorithm had a significant improvement over normal SVM [28] and obtained a little higher AUC score in average than MLMIL [22].

\section{CONCLUSIONS}

A multi-task structured SVM algorithm is developed to leverage both the inter-object correlations and the looselytagged images for achieving more effective training of a large number of inter-related object classifiers. To tackle the issues of multiple and loose tags, an ambiguous image representation algorithm is developed by using bags of instances and a multiple instance learning algorithm is proposed for identifying the positive instances automatically. An object correlation network is constructed for identifying the interrelated learning tasks automatically, and multi-task learning is seamlessly integrated with structured SVM to achieve more effectively training of a large number of inter-related object classifiers. Our experimental results on a large number of object classes have provided very positive results. Our image set and source codes are released on our web site: http://www.cs.uncc.edu/ jfan.

\section{REFERENCES}

[1] Flickr, http://www.flickr.com.

[2] J. Fan, Y. Shen, N. Zhou, Y. Gao, "Harvesting large-scale weakly-tagged image databases from the web", IEEE CVPR, 2010.

[3] MSRC, http://research.microsoft.com/.

[4] Y. Deng, B.S. Manjunath, "Color image segmentation", IEEE CVPR, 1999.

[5] B. Russell, A. Efros, J. Sivic, W. Freeman, A. Zisserman,
"Using multiple segmentations to discover objects and their extent in image collections", IEEE CVPR, 2006.

[6] B.J. Frey, D. Dueck, "Clustering by passing messages between data points", Science, vol.315, 2007

[7] S. Vijayanarasimhan, K. Grauman, "Keywords to visual categories: Multiple-instance learning for weakly supervised object categorization", IEEE CVPR 2008.

[8] Q. Zhang, W. Yu, S. A. Goldman, J. E. Fritts, "Content-based image retrieval using multiple-instance learning", ICML, 2002.

[9] O. Maron, A. L. Ratan, "Multiple-instance learning for natural scene classification", ICML, 1998.

[10] Y. Chen, J. Bi, J. Z. Wang, "MILES: multiple instance learning via embedded instance selection", IEEE Trans. PAMI, vol.28, no.12, pp.1931-1947, 2006.

[11] M. R. Boutell, J. Luo, X. Shen, C.M. Brown,"Learning multi-label scene classification", Pattern Recognition, vol. 37, no.9, pp. 1757-1771, 2004.

[12] Z.-H. Zhu, M.-L. Zhang, "Multi-instance multi-label learning with application to scene classification", NIPS, 2006.

[13] J. Fan, Y. Gao, H. Luo, "Integrating concept ontology and multi-task learning to achieve more effective classifier training for multi-level image annotation", IEEE Trans. on Image Processing, vol. 17, no.3, pp.407-426, 2008.

[14] T. Evgeniou, C.A. Micchelli, M. Pontil, "Learning multiple tasks with kernel methods", Journal of Machine Learning Research, vol.6, pp.615-637, 2005.

[15] S. Kumar, M. Hebert, "Discriminative random fields", Intl. Journal of Computer Vision, vol.68, no.2, pp.179-201, 2006.

[16] J. Yang, Y. Liu, E. X. Ping, A.G. Hauptmann, "Harmonium models for semantic video representation and classification", SIAM Conf. on Data Mining, 2007.

[17] A. Torralba, K. P. Murphy, W. T. Freeman, "Sharing features: efficient boosting procedures for multiclass object detection", IEEE CVPR, 2004.

[18] W. Jiang, S.-F. Chang, A. Loui, "Context-based concept fusion with boosted conditional random fields", IEEE ICASSP, 2007.

[19] J. Tang, X. Hua, M. Wang, Z. Gu, G. Qi, X. Wu, "Correlative linear neighborhood propagation for video annotation", IEEE Trans. on SMC, vol. 39, no.2, pp.409-416, 2009.

[20] J. Liu , M. Li , W.-Y. Ma , Q. Liu , H. Lu, "An adaptive graph model for automatic image annotation", ACM Multimedia Workshop on MIR, 2006.

[21] G.-J. Qi, X.-S. Hua, Y. Rui, J. Tang, T. Mei, H.-J. Zhang, "Correlative multi-label video annotation", ACM Multimedia, pp.17-26, 2007.

[22] Z. Zha, X.-S. Hua, T. Mei, J. Wang, G.-J. Qi, Z. Wang, "Joint multi-label multi-instance learning for image classification", IEEE CVPR, 2008.

[23] I. Tsochantaridis, T. Joachims, T. Hofmann,Y. Altun, "Large margin methods for structured and interdependent output variables", Journal of Machine Learning Research, vol.6, pp.1453-1484, 2005.

[24] T. Joachims, T. Finley, C. Yu, "Cutting-plane training of structural SVMs", Machine Learning, vol. 77, no.1, pp.27-59, 2009.

[25] J.A. Hanley, B.J. Mcneil, "The meaning and use of the area under a receiver operating characteristic (roc) curve", Radiology, vol.143, no.1, pp.29-36, 1982.

[26] J. Fan, Y. Gao, H. Luo, "Multi-level annotation of natural scenes using dominant image components and semantic image concepts", ACM Multimedia, 2004.

[27] H.P. Graf, E. Cosatto, L. Bottou, I. Durdanovic, V. Vapnik, "Parallel support vector machines: The cascade SVM", NIPS 2004.

[28] R. Fan, P. Chen, C.-J. Lin, "Working set selection using the second order information for training SVM", Journal of Machine Learning Research, vol. 6, pp.1889-1918, 2005. 